\title{
GLOBAL LARGE SOLUTIONS TO INITIAL BOUNDARY VALUE PROBLEMS IN ONE-DIMENSIONAL NONLINEAR THERMOVISCOELASTICITY
}

\author{
BY \\ SONG JIANG \\ Institut für Angewandte Mathematik der Universität Bonn, 5300 Bonn 1, Germany
}

\begin{abstract}
Initial boundary value problems in one-dimensional nonlinear thermoviscoelasticity are considered, and the existence of global classical solutions is established by means of the Leray-Schauder fixed point theorem.
\end{abstract}

Introduction. In this paper we study the existence of global smooth solutions to initial boundary value problems in one-dimensional nonlinear thermoviscoelasticity. The conservation laws of mass, momentum, and energy for one-dimensional materials with the reference density $\rho_{0}=1$ are

$$
\begin{gathered}
u_{t}-v_{x}=0 \\
v_{t}-\sigma_{x}=0 \\
\left(e+\frac{v^{2}}{2}\right)_{t}-(\sigma v)_{x}+q_{x}=0,
\end{gathered}
$$

and the second law of thermodynamics is expressed by the Clausius-Duhem inequality

$$
\eta_{t}+\left(\frac{q}{\theta}\right)_{x} \geq 0
$$

where subscripts indicate partial differentiations, $u$ is the deformation gradient, $v$ is the velocity, $e$ denotes the internal energy, $\sigma$ is the stress, $\eta$ stands for the specific entropy, $\theta$ for the temperature, and $q$ for the heat flux.

For one-dimensional, homogeneous, thermoviscoelastic materials, $e, \sigma, \eta$, and $q$ are given by the constitutive relations (see [1])

$$
e=\hat{e}(u, \theta), \quad \sigma=\hat{\sigma}\left(u, \theta, v_{x}\right), \quad \eta=\hat{\eta}(u, \theta), \quad q=\hat{q}\left(u, \theta, \theta_{x}\right),
$$

which in order to be consistent with (1.2), must satisfy

$$
\begin{gathered}
\hat{\sigma}(u, \theta, 0)=\hat{\psi}_{u}(u, \theta), \quad \hat{\eta}(u, \theta)=-\hat{\psi}_{\theta}(u, \theta), \\
(\hat{\sigma}(u, \theta, w)-\hat{\sigma}(u, \theta, 0)) w \geq 0, \quad \hat{q}(u, \theta, g) g \leq 0,
\end{gathered}
$$

where $\psi=e-\theta \eta$ is the Helmholtz free energy function.

Received August 5, 1991 and, in revised form, October 28, 1991.

1991 Mathematics Subject Classification. Primary 35M10, 73C35, 73B30.

Permanent address : Department of Mathematics, Xi'an Jiaotong University, Xi'an, Shaanxi Province, PR China.

E-mail address : unm204@ibm.rhrz.uni-bonn.de. 
Here we consider that the reference configuration is the unit interval $[0,1]$ and that the initial values of the deformation gradient, the velocity, and the temperature are the given functions

$$
u(x, 0)=u_{0}(x), \quad v(x, 0)=v_{0}(x), \quad \theta(x, 0)=\theta_{0}(x), \quad x \in[0,1] .
$$

As boundary conditions we consider

$$
\begin{cases}\sigma(1, t)=-\gamma v(1, t), & \sigma(0, t)=\gamma v(0, t), \quad t \geq 0, \\ \theta(1, t)=\theta(0, t)=T_{0}, & t \geq 0,\end{cases}
$$

where $\gamma=0$ or $\gamma=1$, and $T_{0}>0$ is the reference temperature. The boundary condition (1.6), with $\gamma=1$, boundary damping, represents that the endpoints of the interval $[0,1]$ are connected to some sort of dash pot.

Recently, Dafermos [2], Dafermos and Hsiao [3] considered the following boundary conditions (stress free and thermally insulated):

$$
\sigma(1, t)=\sigma(0, t)=0, \quad q(1, t)=q(0, t)=0, \quad t \geq 0,
$$

and established the existence of global smooth solutions to (1.1), (1.5), (1.7) for a class of solid-like materials by applying the Leray-Schauder fixed point theorem. The techniques in [2] work when only one end of the body is stress-free while the other is fixed, say $v(1, t)=0, \sigma(0, t)=0$. For the initial value problem to (1.1) Zheng and Shen [16] proved the global existence of smooth solutions provided that the initial data are sufficiently small, and Kim [9] established the existence of global weak solutions in $L^{1} \cap B V$. When the material is an ideal gas or a real gas, there are independent investigations on the existence of global classical solutions of (1.1) (see [5-8, 11-14] and the references cited therein).

The purpose of this paper is to show the existence of globally defined classical solutions to (1.1), (1.5), (1.6) for the same class of solid-like materials as in [2]. Our paper is mainly based on techniques in $[2,3]$, subject to the necessary modifications in the proof.

As in [2] we consider the problem of existence of solutions to (1.1), (1.5)-(1.6) for a class of materials with constitutive relations

$$
e=\hat{e}(u, \theta), \quad \sigma=-\hat{p}(u, \theta)+\hat{\mu}(u) v_{x}, \quad q=-\hat{\kappa}(u, \theta) \theta_{x},
$$

where the viscosity $\hat{\mu}(u) u$ satisfies

$$
\hat{\mu}(u) u \geq \mu_{0}>0, \quad 0<u<\infty
$$

for some constant $\mu_{0}$. We assume that $\hat{e}(u, \theta), \hat{p}(u, \theta), \hat{\mu}(u)$, and $\hat{\kappa}(u, \theta)$ are twice continuously differentiable for $0<u<\infty$ and $0 \leq \theta<\infty$, and are interrelated by

$$
\hat{e}_{u}(u, \theta)=-\hat{p}(u, \theta)+\theta \hat{p}_{\theta}(u, \theta)
$$

so as to be consistent with (1.4). Moreover, we will be concerned with solid-like materials, so we require that $\hat{p}(u, \theta)$ be compressive for small $u$ and tensile for large $u$, at any temperature, i.e., there are $0<\tilde{u} \leq \widetilde{U}<\infty$ such that

$$
\begin{aligned}
& \hat{p}(u, \theta) \geq 0, \quad 0<u<\tilde{u}, \quad 0 \leq \theta<\infty, \\
& \hat{p}(u, \theta) \leq 0, \quad \widetilde{U}<u<\infty, \quad 0 \leq \theta<\infty \text {. }
\end{aligned}
$$


Hence, the assumption (1.11) implies that there is a constant $\eta_{0}$ with $\tilde{u} \leq \eta_{0} \leq \tilde{U}$ such that

$$
\hat{p}\left(\eta_{0}, T_{0}\right)=0 .
$$

We impose the following monotone condition on $\hat{p}$ :

$$
\begin{array}{ll}
-\hat{p}_{u}\left(u, T_{0}\right) \geq 0 & \text { for any } \bar{u} \leq u \leq \bar{U} \text { if } \gamma=0 \text { in }(1.6)_{1}, \\
-\hat{p}_{u}\left(u, T_{0}\right) \geq p_{0}>0 & \text { for any } 0<u<\infty \text { if } \gamma=1 \text { in (1.6) } 1 .
\end{array}
$$

Here $p_{0}$ is a constant, and

$$
\begin{aligned}
\bar{u} & :=\widehat{M}^{-1}\left(\min _{\lambda \in[0,1]}\left[\widehat{M}\left(\min \left\{\tilde{u},(1-\lambda) \eta_{0}+\lambda \min _{[0,1]} u_{0}(\cdot)\right\}\right)-2 E_{0}^{1 / 2}(\lambda)\right]-1\right), \\
\bar{U} & :=\widehat{M}^{-1}\left(\max _{\lambda \in[0,1]}\left[\widehat{M}\left(\max \left\{\widetilde{U},(1-\lambda) \eta_{0}+\lambda \max _{[0,1]} u_{0}(\cdot)\right\}\right)+2 E_{0}^{1 / 2}(\lambda)\right]+1\right), \\
E_{0}(\lambda):= & \left(1+\frac{2 \gamma^{2}}{p_{0}}\right) \int_{0}^{1}\left\{E\left((1-\lambda) \eta_{0}+\lambda u_{0}(x),(1-\lambda) T_{0}+\lambda \theta_{0}(x)\right)+\frac{\lambda^{2} v_{0}^{2}(x)}{2}\right\} d x \\
& \quad+\gamma^{2} \eta_{0}^{2}, \quad E(u, \theta):=\hat{\psi}(u, \theta)-\hat{\psi}\left(\eta_{0}, T_{0}\right)-\left(\theta-T_{0}\right) \hat{\psi}_{\theta}(u, \theta),
\end{aligned}
$$

and $\hat{\psi}(u, \theta)$ is the Helmholtz free energy function. In view of (1.9), $\widehat{M}(u)$ is a strictly increasing function that maps $(0, \infty)$ onto $(-\infty, \infty)$. Using $(1.11)$ we can show that $u$ is a priori bounded, $\bar{u}<u(x, t)<\bar{U}$ (cf. Lemma 2.3), and hence no restrictions are necessary on the behavior of $\hat{e}(u, \theta), \hat{p}(u, \theta)$, and $\hat{\kappa}(u, \theta)$ at $u=0+$ and $u=\infty$. As concerns the temperature, we impose the following growth conditions upon $\hat{e}(u, \theta), \hat{p}(u, \theta)$, and $\hat{\kappa}(u, \theta)$. There are positive constants $\nu$ and $N$ possibly depending on $\bar{u}$ and/or $\bar{U}$ such that for any $\bar{u} \leq u \leq \bar{U}, 0 \leq \theta<\infty$,

$$
\begin{gathered}
\hat{e}(u, 0) \geq 0, \quad \nu \leq \hat{e}_{\theta}(u, \theta) \leq N\left(1+\theta^{1 / 3}\right), \\
\left|\hat{p}_{u}(u, \theta)\right| \leq N\left(1+\theta^{4 / 3}\right), \quad\left|\hat{p}_{\theta}(u, \theta)\right| \leq N\left(1+\theta^{1 / 3}\right), \\
\left.\nu \leq \hat{\kappa}(u, \theta) \leq N, \quad\left|\hat{\kappa}_{u}(u, \theta)\right| \leq N, \quad\left|\hat{\kappa}_{\theta}(u, \theta)\right| \leq N, \quad\left|\hat{\kappa}_{u u}(u, \theta)\right| \leq N .15\right)
\end{gathered}
$$

We use the familiar notations $C^{\alpha}[0,1]$ for the Banach space of functions on $[0,1]$ that are uniformly Hölder continuous with exponent $\alpha$ and $C^{\alpha, \alpha / 2}\left(Q_{T}\right)$ for those functions on $Q_{T}:=[0,1] \times[0, T]$ that are uniformly Hölder continuous with exponent $\alpha$ in $x$ and $\alpha / 2$ in $t$. The norms of $C^{\alpha}[0,1]$ and $C^{\alpha, \alpha / 2}\left(Q_{T}\right)$ will be denoted by $\|\cdot\|_{\alpha}$ and $\|\cdot\| \|_{\alpha}$, respectively. The main result of this paper is

THEOREM 1.1. Let $u_{0}(x), u_{0}^{\prime}(x), v_{0}(x), v_{0}^{\prime}(x), v_{0}^{\prime \prime}(x), \theta_{0}(x), \theta_{0}^{\prime}(x)$, and $\theta_{0}^{\prime \prime}(x)$ be in $C^{\alpha}[0,1]$ for some $\alpha \in(0,1)$. Let $u_{0}(x)>0, \theta_{0}(x)>0$ for $x \in[0,1]$, and assume that the initial data are compatible with the boundary conditions (1.6). Then 
there exists a unique solution $\{u(x, t), v(x, t), \theta(x, t)\}$ to (1.1), (1.5), (1.6) on $[0,1] \times[0, \infty)$ such that for every $T>0$ the functions $u, u_{x}, u_{t}, u_{x t}, v, v_{x}, v_{t}$, $v_{x x}, \theta, \theta_{x}, \theta_{t}, \theta_{x x}$ are in $C^{\alpha, \alpha / 2}\left(Q_{T}\right)$ and $u_{t t}, v_{x t}, \theta_{x t}$ are in $L^{2}\left(Q_{T}\right)$. Furthermore, $\theta(x, t)>0, \bar{u}<u(x, t)<\bar{U}$ for $0 \leq x \leq 1,0 \leq t<\infty$, where $\bar{u}, \bar{U}$ are the same as in (1.14).

We will apply the Leray-Schauder fixed point theorem to prove the theorem in Sec.

3. Section 2 is devoted to the necessary a priori estimates.

REMARK 1.1. The techniques in this paper work for the boundary conditions:

$$
\begin{aligned}
& \sigma(1, t)=-v(1, t), \quad \sigma(0, t)=v(0, t), \quad t \geq 0, \\
& q(1, t)=q(0, t)=0, \quad t \geq 0
\end{aligned}
$$

and for the boundary conditions that one end of the interval $[0,1]$ is stress-free while the other has the boundary damping, say $\sigma(1, t)=0, \sigma(0, t)=v(0, t)$, and an identical existence theorem can be obtained.

REMARK 1.2. The slower the growth rate of $\hat{e}(u, \theta)$, the faster the growth rate of $\hat{p}(u, \theta)$ that can be treated by the same procedure here. Furthermore, if the specific heat and/or the heat conductivity grows with the temperature, say $\hat{e}_{\theta}(u, \theta) \geq$ $\nu\left(1+\theta^{s}\right)$ and/or $\hat{\kappa}(u, \theta) \geq \nu\left(1+\theta^{s}\right)$, then higher growth rates of $\hat{e}(u, \theta)$ and $\hat{p}(u, \theta)$ may be tolerated.

2. A priori estimates. Let $T>0$ be arbitrary but fixed. Throughout this paper, $\Lambda$ will denote a generic constant which may depend at most on $T, T_{0}, \nu, \mu_{0}, N, \bar{u}, \bar{U}$, and upper bounds of the $C^{\alpha}[0,1]$ norm of $u_{0}, u_{0}^{\prime}, v_{0}, v_{0}^{\prime}, v_{0}^{\prime \prime}, \theta_{0}, \theta_{0}^{\prime}$, and $\theta_{0}^{\prime \prime}$. Our aim in this section is to show the following.

THEOREM 2.1. Let $\{u(x, t), v(x, t), \theta(x, t)\}$ be a solution of $(1.1),(1.5),(1.6)$ on $[0,1] \times[0, \infty)$ in the function class indicated in Theorem 1.1. Then $u, v, v_{x}, \theta, \theta_{x}$ can be a priori bounded in $C^{1 / 3,1 / 6}\left(Q_{T}\right)$, i.e.,

$$
\|\| u\left\|_{1 / 3}+\right\|\|v\|_{1 / 3}+\left\|\left|v_{x}\left\|_{1 / 3}+\right\|\right| \theta\right\|_{1 / 3}+\left\|\theta_{x}\right\|_{1 / 3} \leq \Lambda .
$$

Furthermore, $\theta(t, x)>0, \bar{u}<u(t, x)<\bar{U}$ for any $x \in[0,1]$ and $t \geq 0$.

The proof of Theorem 2.1 is broken into a sequence of lemmas. The first observation is that using $\sigma$ in (1.8) we can write $(1.1)_{2}$ in the form

$$
v_{t}+\hat{p}(u, \theta)_{x}=\left(\hat{\mu}(u) v_{x}\right)_{x},
$$

and utilizing $(1.1)_{3},(1.1)_{2},(1.10),(1.8)$, and $(1.1)_{1}$ we have

$$
\hat{e}_{\theta}(u, \theta) \theta_{t}+\theta \hat{p}_{\theta}(u, \theta) v_{x}-\hat{\mu}(u) v_{x}^{2}=\left(\hat{\kappa}(u, \theta) \theta_{x}\right)_{x} .
$$

If we apply the maximum principle [15, III.3] to (2.2), recalling $\theta_{0}(x)>0$, we infer LEMMA 2.1.

$$
\theta(x, t)>0 \text { for } 0 \leq x \leq 1,0 \leq t<\infty .
$$

Now we derive an estimate on $u, v$, and $\theta$ by exploiting some relations associated with the second law of thermodynamics. 
Lemma 2.2. If $\bar{u} \leq u(t, x) \leq \bar{U}$ for all $x \in[0,1]$ and $t \in[0, \tau], \tau>0$, then

$$
\begin{gathered}
\frac{\nu}{2} \int_{0}^{1} \frac{\left(\theta(x, t)-T_{0}\right)^{2}}{\theta(x, t)+T_{0}} d x+\frac{\omega_{\gamma}}{2} \int_{0}^{1}\left(u(x, t)-\eta_{0}\right)^{2} d x+\frac{1}{2} \int_{0}^{1} v^{2}(x, t) d x \\
\leq \int_{0}^{1}\left(E\left(u_{0}(x), \theta_{0}(x)\right)+\frac{v_{0}^{2}(x)}{2}\right) d x \equiv e_{0}, \quad \forall 0 \leq t \leq \tau,
\end{gathered}
$$

where $\omega_{\gamma}=0$ for $\gamma=0$ and $\omega_{\gamma}=p_{0}$ for $\gamma=1, E(u, \theta)$ and $p_{0}$ are the same as in $(1.14)_{4}$ and in $(1.13)_{2}$, respectively.

Proof. Recalling the definition $(1.14)_{4}$ of $E(u, \theta)$, making use of $(1.1)_{1},(1.1)_{2}$, (2.2), and (1.4), noting that $-\hat{p}(u, \theta)=\hat{\psi}_{u}(u, \theta)$ and $\hat{e}_{\theta}(u, \theta)=-\theta \partial^{2} \hat{\psi}(u, \theta) / \partial \theta^{2}$, we obtain after a calculation that

$$
\partial_{t}\left(E(u, \theta)+\frac{v^{2}}{2}\right)+\frac{T_{0} \hat{\mu}(u)}{\theta} v_{x}^{2}+\frac{T_{0} \hat{\kappa}(u, \theta)}{\theta^{2}} \theta_{x}^{2}=\partial_{x}\left(\sigma v+\frac{\left(\theta-T_{0}\right)}{\theta} \hat{\kappa}(u, \theta) \theta_{x}\right) .
$$

We integrate (2.4) over $[0,1] \times[0, t](0 \leq t \leq T)$ and apply the boundary conditions (1.6) to arrive at

$$
\begin{gathered}
\int_{0}^{1}\left(E(u, \theta)+\frac{v^{2}}{2}\right)(x, t) d x+T_{0} \int_{0}^{t} \int_{0}^{1}\left(\frac{\hat{\mu}(u)}{\theta} v_{x}^{2}+\frac{\hat{\kappa}(u, \theta)}{\theta^{2}} \theta_{x}^{2}\right) d x d s \\
\quad+\gamma \int_{0}^{t}\left(v^{2}(1, s)+v^{2}(0, s)\right) d s=\int_{0}^{1}\left(E\left(u_{0}(x), \theta_{0}(x)\right)+\frac{v_{0}^{2}(x)}{2}\right) d x .
\end{gathered}
$$

Recalling $\hat{\psi}_{u u}=-\hat{p}_{u}$, if we use the mean value theorem (or the Taylor theorem), (1.15), and (1.12)-(1.13), we see that

$$
E(u, \theta)-\hat{\psi}\left(u, T_{0}\right)+\hat{\psi}\left(\eta_{0}, T_{0}\right) \geq \frac{\nu}{2} \frac{\left(\theta-T_{0}\right)^{2}}{\left(\theta+T_{0}\right)}
$$

and

$$
\hat{\psi}\left(u, T_{0}\right)-\hat{\psi}\left(\eta_{0}, T_{0}\right) \geq \frac{\omega_{\gamma}}{2}\left(u-\eta_{0}\right)^{2}
$$

for $\bar{u} \leq u \leq \bar{U}$, which adding to (2.6) gives

$$
E(u, \theta) \geq \frac{\nu}{2} \frac{\left(\theta-T_{0}\right)^{2}}{\left(\theta+T_{0}\right)}+\frac{\omega_{\gamma}}{2}\left(u-\eta_{0}\right)^{2} \text { for } \bar{u} \leq u \leq \bar{U} .
$$

Inserting (2.7) into (2.5) yields the lemma.

Next, we want to bound the deformation gradient $u(x, t)$. To this end we rewrite (2.1), using $(1.14)_{4}$, as follows:

$$
v_{t}+\hat{p}(u, \theta)_{x}=\widehat{M}(u)_{t x} .
$$

Lemma 2.3. We have

$$
\bar{u}<u(x, t)<\bar{U}, \quad 0 \leq x \leq 1, \quad 0 \leq t<\infty,
$$

where $\bar{u}$ and $\bar{U}$ are defined by $(1.14)_{1}$ and $(1.14)_{2}$, respectively. 
Proof. If we integrate $(2.8)$ over $[0, y] \times[s, \tau]$ and $[y, 1] \times[s, \tau], 0 \leq y \leq 1$, $0 \leq s<\tau$, respectively, and apply the boundary condition (1.6) $)_{1}$, we obtain $\widehat{M}(u(y, \tau))-\widehat{M}(u(y, s))=\int_{s}^{\tau} p(y, t) d t+\gamma \int_{s}^{\tau} v(0, t) d t+\int_{0}^{y}(v(x, \tau)-v(x, s)) d x$

and

$\widehat{M}(u(y, \tau))-\widehat{M}(u(y, s))=\int_{s}^{\tau} p(y, t) d t-\gamma \int_{s}^{\tau} v(1, t) d t-\int_{y}^{1}(v(x, \tau)-v(x, s)) d x$,

where $p(y, t)=\hat{p}(u(y, t), \theta(y, t))$. We add (2.11) to (2.10) and take $u_{t}=v_{x}$ into account to deduce

$$
\begin{aligned}
\widehat{M}(u(y, \tau))-\widehat{M}(u(y, s))= & \int_{s}^{\tau} p(y, t) d t-\frac{\gamma}{2} \int_{s}^{\tau} \int_{0}^{1} v_{x}(x, t) d x d t \\
& +\frac{1}{2}\left(\int_{0}^{y}-\int_{y}^{1}\right)(v(x, \tau)-v(x, s)) d x \\
= & \int_{s}^{\tau} p(y, t) d t-\frac{\gamma}{2} \int_{0}^{1}(u(x, \tau)-u(x, s)) d x \\
& +\frac{1}{2}\left(\int_{0}^{y}-\int_{y}^{1}\right)(v(x, \tau)-v(x, s)) d x .
\end{aligned}
$$

By Lemma 2.2 and the Schwarz inequality, recalling the definition $(1.14)_{3}$, we see that if $\bar{u} \leq u(x, t) \leq \bar{U}$ for $0 \leq x \leq 1,0 \leq t \leq \tau$, then we have

$$
\begin{aligned}
& \frac{\gamma}{2}\left|\int_{0}^{1}(u(x, \tau)-u(x, s)) d x\right|+\frac{1}{2}\left|\left(\int_{0}^{y}-\int_{y}^{1}\right)(v(\tau, x)-v(s, x)) d x\right| \\
& \quad \leq \gamma \max _{[0, \tau]}\left(\int_{0}^{1} u^{2}(x, \cdot) d x\right)^{1 / 2}+\max _{[0, \tau]}\left(\int_{0}^{1} v^{2}(x, \cdot) d x\right)^{1 / 2} \\
& \leq \gamma\left(2 \eta_{0}^{2}+4 e_{0} / p_{0}\right)^{1 / 2}+\left(2 e_{0}\right)^{1 / 2} \leq 2\left(\left(1+2 \gamma^{2} / p_{0}\right) e_{0}+\gamma^{2} \eta_{0}^{2}\right)^{1 / 2} \equiv 2 E_{0}^{1 / 2}(1) .
\end{aligned}
$$

In particular, $(1.14)_{1}$ and $(1.14)_{2}$ yield $\bar{u}<u_{0}(x)<\bar{U}, 0 \leq x \leq 1$. Thus, if $\bar{u}<u(x, t)<\bar{U}$ is violated on $[0,1] \times[0, \infty)$, then there are $\tau>0$ and $y \in[0,1]$ such that

$$
\bar{u}<u(x, t)<\bar{U} \quad \text { for } x \in[0,1], 0 \leq t<\tau, \quad \text { but } u(y, \tau)=\bar{u} \quad \text { or } \quad u(y, \tau)=\bar{U} .
$$

Note that $\bar{u}<\tilde{u}$. If $u(y, \tau)=\bar{u}$, then either $u(y, t)<\tilde{u}$ for $0 \leq t \leq \tau$, or $u(y, t)<\tilde{u}$ for $0 \leq s<t \leq \tau$, but $u(y, s)=\tilde{u}$. Recalling that, on account of (2.14), $\bar{u} \leq u(x, t) \leq \bar{U}$ for $0 \leq x \leq 1$ and $0 \leq t \leq \tau$, in the former case we apply (2.12) with $s=0$ and utilize (1.11) and (2.13) to deduce

$$
\widehat{M}(u(y, \tau))>\widehat{M}\left(u_{0}(y)\right)-2 E_{0}^{1 / 2}(1)-1,
$$

while in the latter case (2.12) combined with (1.11) and (2.13) implies

$$
\widehat{M}(u(y, \tau))>\widehat{M}(\tilde{u})-2 E_{0}^{1 / 2}(1)-1 .
$$


In either case, by $(1.14)_{1}, \widehat{M}(u(y, \tau))>\widehat{M}(\bar{u})$ which contradicts $u(y, \tau)=\bar{u}$. Hence, $\bar{u}<u(t, x), 0 \leq x \leq 1,0 \leq t<\infty$.

Similarly, we can show that $u(y, \tau)=\bar{U}$ is a contradiction. This shows $u(t, x)<$ $\bar{U}$ for $0 \leq x \leq 1,0 \leq t<\infty$.

As a result of Lemmas $2.1-2.3$ one gets

$$
\max _{[0, T]} \int_{0}^{1} \theta(x, t) d x \leq \Lambda \text {. }
$$

By (1.15) and Lemma 2.1 we find that $\int_{0}^{1} e(x, t) d x \geq 0$. Using this fact, we integrate $(1.1)_{3}$ over $[0,1] \times[0, T]$ and apply the boundary condition $(1.6)_{1}$ to infer

$$
\int_{0}^{T}(q(1, t)-q(0, t)) d t \leq \int_{0}^{1}\left(\hat{e}\left(u_{0}(x), \theta_{0}(x)\right)+\frac{v_{0}^{2}}{2}\right)(x) d x \leq \Lambda,
$$

where $q(x, t)=-\hat{\kappa}(u(x, t), \theta(x, t)) \theta_{x}(x, t)$.

We now proceed to get estimates. We have

LEMMA 2.4 .

$$
\begin{gathered}
\int_{0}^{T} \int_{0}^{1} \theta^{-4 / 3} \theta_{x}^{2} d x d t \leq \Lambda, \\
\int_{0}^{T} \int_{0}^{1} \theta^{8 / 3} d x d t \leq \Lambda, \\
\int_{0}^{T} \max _{[0,1]} \theta^{5 / 3}(\cdot, t) d t \leq \Lambda .
\end{gathered}
$$

Proof. Define $\widehat{H}(u, \theta):=\int_{0}^{\theta} \xi^{-1 / 3} \hat{e}_{\theta}(u, \xi) d \xi$. By (1.15) and (1.10),

$$
\begin{gathered}
|\widehat{H}(u, \theta)| \leq 2 N(1+\theta), \quad \widehat{H}_{\theta}(u, \theta)=\theta^{-1 / 3} \hat{e}_{\theta}(u, \theta), \\
\widehat{H}_{u}(u, \theta)=\theta^{2 / 3} \hat{p}_{\theta}(u, \theta)-\widehat{G}(u, \theta),
\end{gathered}
$$

where

$$
\widehat{G}(u, \theta):=\frac{2}{3} \int_{0}^{\theta} \xi^{-1 / 3} \hat{p}_{\theta}(u, \xi) d \xi .
$$

Multiplication of (2.2) with $\theta^{-1 / 3}$ and use of (2.22)-(2.23) yield

$$
H_{t}+\widehat{G}(u, \theta) v_{x}-\theta^{-1 / 3} \hat{\mu}(u) v_{x}^{2}-\theta^{-1 / 3}\left(\hat{\kappa}(u, \theta) \theta_{x}\right)_{x}=0 .
$$

Here $H(x, t):=\widehat{H}(u(x, t), \theta(x, t))$. In view of (2.23) and (1.16), $|\widehat{G}(u, \theta)| \leq$ $2 N(1+\theta)$. So if we integrate $(2.24)$ over $[0,1] \times[0, T]$, integrate by parts with respect to $x$, and utilize $(1.6)_{2},(1.9)$, Lemma $2.3,(1.17),(2.18),(2.22),(2.17)$, and the Schwarz inequality, we arrive at

$$
\begin{aligned}
\mu_{0} \bar{U}^{-1} \int_{0}^{T} \int_{0}^{1} \theta^{-1 / 3} v_{x}^{2} d x d t+\frac{\nu}{3} \int_{0}^{T} \int_{0}^{1} \theta^{-4 / 3} \theta_{x}^{2} d x d t \\
\quad \leq \Lambda+\int_{0}^{T} \int_{0}^{1} \widehat{G}(u, \theta) v_{x} d x d t+T_{0}^{-1 / 3} \int_{0}^{T}(q(1, t)-q(0, t)) d t \\
\quad \leq \Lambda+4 N^{2} \bar{U} \mu_{0}^{-1} \int_{0}^{T} \int_{0}^{1} \theta^{7 / 3} d x d t+\frac{1}{2} \mu_{0} \bar{U}^{-1} \int_{0}^{T} \int_{0}^{1} \theta^{-1 / 3} v_{x}^{2} d x d t
\end{aligned}
$$


By (2.17), the Sobolev imbedding theorem $W^{1,1} \hookrightarrow L^{\infty}$, and the inequality $a b \leq$ $a^{p} / p+b^{q} / q(a, b \geq 0, p, q>1,1 / p+1 / q=1)$, we find

$$
\begin{aligned}
\int_{0}^{T} \int_{0}^{1} \theta^{8 / 3} d x d t & \leq \Lambda \int_{0}^{T} \max _{[0,1]} \theta^{5 / 3}(\cdot, t) d t \\
& \leq \Lambda \int_{0}^{T} \int_{0}^{1}\left(\theta^{5 / 3}+\theta^{2 / 3}\left|\theta_{x}\right|\right) d x d t \\
& \leq \Lambda+\frac{1}{2} \int_{0}^{T} \int_{0}^{1} \theta^{8 / 3} d x d t+\Lambda \int_{0}^{T} \int_{0}^{1} \theta^{-4 / 3} \theta_{x}^{2} d x d t .
\end{aligned}
$$

This gives

$$
\begin{aligned}
4 N^{2} \bar{U} \mu_{0}^{-1} \int_{0}^{t} \int_{0}^{1} \theta^{7 / 3} d x d t & \leq \Lambda+\frac{\nu}{12 \Lambda} \int_{0}^{T} \int_{0}^{1} \theta^{8 / 3} d x d t \\
& \leq \Lambda+\frac{\nu}{6} \int_{0}^{T} \int_{0}^{1} \theta^{-4 / 3} \theta_{x}^{2} d x d t
\end{aligned}
$$

which together with (2.25) proves (2.19). (2.26) thus implies (2.20) and (2.21).

The following lemma can be proved by multiplying (2.1) with $v$, integrating over $[0,1] \times[0, T]$, and using Lemma 2.4; its proof is the same as in [2, Lemma 2.2] and we shall not repeat it here.

LEMMA 2.5 .

$$
\int_{0}^{T} \int_{0}^{1} v_{x}^{2}(x, t) d x d t \leq \Lambda .
$$

In a sequel we establish the higher-order estimates of solutions. Following [2], we define

$$
Y:=\max _{[0, T]} \int_{0}^{1} \theta_{x}^{2}(x, t) d x, \quad Z:=\max _{[0, T]} \int_{0}^{1} v_{x x}^{2}(x, t) d x .
$$

It follows from the Sobolev imbedding theorem $W^{1,1} \hookrightarrow L^{\infty},(2.17)$, and the Schwarz inequality that

$$
\begin{aligned}
\theta^{3 / 2}(y, t) & \leq \Lambda\left(\max _{Q_{T}} \theta\right)^{1 / 2}+\int_{0}^{1} \theta^{1 / 2}(x, t)\left|\theta_{x}(x, t)\right| d x \\
& \leq \Lambda+\frac{1}{2}\left(\max _{Q_{T}} \theta\right)^{3 / 2}+\Lambda Y^{1 / 2}, \quad \forall 0 \leq y \leq 1,0 \leq t \leq T,
\end{aligned}
$$

which yields

$$
\max _{Q_{T}} \theta(x, t) \leq \Lambda+\Lambda Y^{1 / 3} .
$$

By virtue of the interpolation inequality and Lemma 2.2 one has

$$
\begin{aligned}
\int_{0}^{1} v_{x}^{2}(x, t) d x & \leq \Lambda \int_{0}^{1} v^{2}(x, t) d x+\Lambda\left(\int_{0}^{1} v^{2}(x, t) d x\right)^{1 / 2}\left(\int_{0}^{1} v_{x x}^{2}(x, t) d x\right)^{1 / 2} \\
& \leq \Lambda Z^{1 / 2}+\Lambda, \quad \forall t \in[0, T]
\end{aligned}
$$


which in conjunction with the Sobolev imbedding theorem and the Schwarz inequality implies

$$
\begin{aligned}
v_{x}^{2}(x, t) & \leq \Lambda\left(\int_{0}^{1} v_{x}^{2}(y, t) d y+\int_{0}^{1}\left|v_{x}(y, t)\right|\left|v_{x x}(y, t)\right| d y\right) \\
& \leq \Lambda+\Lambda Z^{3 / 4}, \quad \forall x \in[0,1], t \in[0, T] .
\end{aligned}
$$

Therefore,

$$
\max _{Q_{T}}\left|v_{x}(x, t)\right| \leq \Lambda+\Lambda Z^{3 / 8}
$$

LEMMA 2.6.

$$
\max _{[0, T]} \int_{0}^{1} u_{x}^{2}(x, t) d x \leq \Lambda+\Lambda Y^{1 / 9} .
$$

The proof of Lemma 2.6 is completely the same as that of Lemma 2.3 in [2]. So, we omit its proof here.

LEMMA 2.7.

$$
\begin{gathered}
Y \leq \Lambda+\Lambda Z^{3 / 4} \\
\int_{0}^{T} \int_{0}^{1} \theta_{t}^{2}(x, t) d x d t \leq \Lambda+\Lambda Z^{3 / 4}
\end{gathered}
$$

Proof. Let

$$
\widehat{Q}(u, \theta):=\int_{0}^{\theta} \hat{\kappa}(u, \xi) d \xi .
$$

Setting $Q(x, t)=\widehat{Q}(u(x, t), \theta(x, t))$, we multiply (2.2) by $Q_{t}$, integrate over $[0,1] \times[0, t], 0 \leq t \leq T$, and integrate by parts with respect to $x$ to get

$$
\begin{gathered}
\int_{0}^{t} \int_{0}^{1}\left(\hat{e}_{\theta} \theta_{t}+\theta \hat{p}_{\theta} v_{x}-\hat{\mu} v_{x}^{2}\right) Q_{t} d x d \tau+\int_{0}^{t} \int_{0}^{1} \hat{\kappa} \theta_{x} Q_{x t} d x d \tau \\
-\int_{0}^{t}\left(\hat{\kappa} \theta_{x} Q_{t}\right)(1, \tau) d \tau+\int_{0}^{t}\left(\hat{\kappa} \theta_{x} Q_{t}\right)(0, \tau) d \tau=0 .
\end{gathered}
$$

We now have to estimate every term in (2.36). Note that

$$
Q_{t}=\widehat{Q}_{u} v_{x}+\hat{\kappa} \theta_{t}, \quad Q_{x t}=\left(\hat{\kappa} \theta_{x}\right)_{t}+\widehat{Q}_{u} v_{x x}+\widehat{Q}_{u u} v_{x} u_{x}+\hat{\kappa}_{u} u_{x} \theta_{t} .
$$

It follows from the fact that, in view of (1.17), $\left|\widehat{Q}_{u}\right|,\left|\widehat{Q}_{u u}\right| \leq N \theta$, the assumptions (1.15) and (1.17), (2.31), and (2.20) that

$$
\begin{aligned}
\int_{0}^{t} \int_{0}^{1} \hat{e}_{\theta} \theta_{t} Q_{t} d x d \tau & \geq \nu^{2} \int_{0}^{t} \int_{0}^{1} \theta_{t}^{2} d x d \tau-\left|\int_{0}^{t} \int_{0}^{1} \hat{e}_{\theta} \theta_{t} \widehat{Q}_{u} v_{x} d x d \tau\right| \\
& \geq \frac{\nu^{2}}{2} \int_{0}^{t} \int_{0}^{1} \theta_{t}^{2} d x d \tau-\Lambda \max _{Q_{T}} v_{x}^{2} \int_{0}^{t} \int_{0}^{1}\left(1+\theta^{8 / 3}\right) d x d \tau \\
& \geq \frac{\nu^{2}}{2} \int_{0}^{t} \int_{0}^{1} \theta_{t}^{2} d x d \tau-\left(\Lambda+\Lambda Z^{3 / 4}\right)
\end{aligned}
$$


Making use of the inequality $a b \leq a^{p} / p+b^{q} / q(a, b \geq 0 ; p, q>1,1 / p+1 / q=1)$ and following the same arguments as those used for (2.51)-(2.53) in [2], we obtain

$$
\int_{0}^{t} \int_{0}^{1} \hat{\kappa} \theta_{x}\left(\hat{\kappa} \theta_{x}\right)_{t} d x d \tau \geq \frac{\nu^{2}}{2} \int_{0}^{1} \theta_{x}^{2}(x, t) d x-\Lambda
$$

and

$$
\begin{gathered}
\left|\int_{0}^{t} \int_{0}^{1}\left(\theta \hat{p}_{\theta} v_{x}-\hat{\mu} v_{x}^{2}\right) \widehat{Q}_{u} v_{x} d x d \tau\right|+\left|\int_{0}^{t} \int_{0}^{1}\left(\theta \hat{p}_{\theta} v_{x}-\hat{\mu} v_{x}^{2}\right) \hat{\kappa} \theta_{t} d x d \tau\right| \\
+\left|\int_{0}^{t} \int_{0}^{1} \hat{\kappa} \theta_{x} \widehat{Q}_{u} v_{x x} d x d \tau\right|+\left|\int_{0}^{t} \int_{0}^{1} \hat{\kappa} \theta_{x} \widehat{Q}_{u u} v_{x} u_{x} d x d \tau\right| \\
\leq \Lambda+\Lambda Z^{3 / 4}+\frac{\nu^{2}}{16} \int_{0}^{t} \int_{0}^{1} \theta_{t}^{2} d x d \tau+\frac{\nu^{2}}{12} Y .
\end{gathered}
$$

By the Schwarz inequality,

$$
\begin{gathered}
\left|\int_{0}^{t} \int_{0}^{1} \hat{\kappa} \theta_{x} \hat{\kappa}_{u} u_{x} \theta_{t} d x d \tau\right| \leq \frac{\nu^{2}}{16} \int_{0}^{t} \int_{0}^{1} \theta_{t}^{2} d x d \tau+\Lambda \int_{0}^{t} \int_{0}^{1}\left(\hat{\kappa} \theta_{x}\right)^{2} u_{x}^{2} d x d \tau \\
\leq \frac{\nu^{2}}{16} \int_{0}^{t} \int_{0}^{1} \theta_{t}^{2} d x d \tau+\Lambda+\frac{\nu^{2}}{24} Y+\Lambda\left(\int_{0}^{t} \int_{0}^{1}\left[\left(\hat{\kappa} \theta_{x}\right)_{x}\right]^{2} d x d \tau\right)^{3 / 4}
\end{gathered}
$$

where we have used the following estimate, which follows from the Sobolev imbedding theorem $W^{1,1} \hookrightarrow L^{\infty}$, Lemma 2.6, (2.19), and (2.30):

$\Lambda \int_{0}^{t} \int_{0}^{1}\left(\hat{\kappa} \theta_{x}\right)^{2} u_{x}^{2} d x d \tau \leq \Lambda \int_{0}^{t} \max _{[0,1]}\left(\hat{\kappa} \theta_{x}\right)^{2} \int_{0}^{1} u_{x}^{2} d x d \tau$

$$
\begin{aligned}
& \leq\left(\Lambda+\Lambda Y^{1 / 9}\right)\left(\int_{0}^{t} \int_{0}^{1}\left(\hat{\kappa} \theta_{x}\right)^{2}+\int_{0}^{t} \int_{0}^{1}\left|\hat{\kappa} \theta_{x}\right|\left|\left(\hat{\kappa} \theta_{x}\right)_{x}\right| d x d \tau\right) \\
& \leq\left(\Lambda+\Lambda Y^{1 / 9}\right)\left\{\Lambda \max _{Q_{T}} \theta^{4 / 3}+\max _{Q_{T}} \theta^{2 / 3}\left(\int_{0}^{t} \int_{0}^{1} \theta^{-4 / 3} \theta_{x}^{2}\right)^{1 / 2}\left(\int_{0}^{t} \int_{0}^{1}\left[\left(\hat{\kappa} \theta_{x}\right)_{x}\right]^{2}\right)^{1 / 2}\right\} \\
& \leq \Lambda+\frac{\nu^{2}}{24} Y+\Lambda\left(\int_{0}^{t} \int_{0}^{1}\left[\left(\hat{\kappa} \theta_{x}\right)_{x}\right]^{2} d x d \tau\right)^{3 / 4} .
\end{aligned}
$$

We now estimate the boundary terms in (2.36). Let $\zeta=1$ or $\zeta=0$. In view of (2.9) and (1.6) $)_{2}$, we have that $\left|\widehat{Q}_{u}(u(\zeta, t), \theta(\zeta, t))\right| \leq \Lambda$. So by virtue of $(1.6)_{2},(2.37),(2.31),(2.19)$, and (2.30), we find that (cf. the proof of $(2.42)$ )

$$
\begin{aligned}
\left|\int_{0}^{t}\left(\hat{\kappa} \theta_{x} Q_{t}\right)(\zeta, \tau) d \tau\right| & =\left|\int_{0}^{t}\left(\hat{\kappa} \theta_{x} \widehat{Q}_{u} v_{x}\right)(\zeta, \tau) d \tau\right| \\
& \leq \Lambda \max _{Q_{T}}\left|v_{x}\right|^{2}+\Lambda \int_{0}^{t} \max _{[0,1]}\left(\hat{\kappa} \theta_{x}\right)^{2} d \tau \\
& \leq \Lambda+\Lambda Z^{3 / 4}+\frac{\nu^{2}}{40} Y+\Lambda\left(\int_{0}^{t} \int_{0}^{1}\left[\left(\hat{\kappa} \theta_{x}\right)_{x}\right]^{2} d x d \tau\right)^{3 / 4} .
\end{aligned}
$$


Note that by (2.30)-(2.31), (2.20), and Lemma 2.5,

$$
\begin{gathered}
\int_{0}^{t} \int_{0}^{1} \hat{e}_{\theta}^{2} \theta_{t}^{2} d x d \tau+\int_{0}^{t} \int_{0}^{1}\left(\theta \hat{p}_{\theta} v_{x}-\hat{\mu} v_{x}^{2}\right)^{2} d x d \tau \\
\leq\left(\Lambda+\Lambda Y^{2 / 9}\right) \int_{0}^{t} \int_{0}^{1} \theta_{t}^{2} d x d \tau+\Lambda+\Lambda Z^{3 / 4}
\end{gathered}
$$

which together with $(2.2)$ gives

$$
\Lambda\left(\int_{0}^{t} \int_{0}^{1}\left[\left(\hat{\kappa} \theta_{x}\right)_{x}\right]^{2} d x d \tau\right)^{3 / 4} \leq \Lambda+\frac{\nu^{2}}{40} Y+\frac{\nu^{2}}{24} \int_{0}^{t} \int_{0}^{1} \theta_{t}^{2} d x d \tau+\Lambda Z^{3 / 4}
$$

Combining (2.36)-(2.41) and (2.43)-(2.44), we get

$$
\frac{\nu^{2}}{4} \int_{0}^{t} \int_{0}^{1} \theta_{t}^{2} d x d \tau+\frac{\nu^{2}}{2} \int_{0}^{1} \theta_{x}^{2}(x, t) d x \leq \Lambda+\Lambda Z^{3 / 4}+\frac{\nu^{2}}{4} Y, \quad \forall t \in[0, T],
$$

which shows (2.33) and (2.34).

Differentiate $(2.1)$ formally with respect to $t$, multiply by $v_{t}$, and integrate over $[0,1] \times[0, t], t \in(0, T]$. Integrating by parts with respect to $x$, we infer by the same procedure as in [2, Lemma 2.5$]$ that

LEMMA 2.8.

$$
\max _{[0, T]} \int_{0}^{1} v_{t}^{2}(x, t) d x+\int_{0}^{T} \int_{0}^{1} v_{x t}^{2} d x d t \leq \Lambda+\Lambda Z^{11 / 12} .
$$

The proof of the following lemma can be found in [2, Lemma 2.6] and will thus be omitted here.

LEMMA 2.9.

$$
\max _{[0, T]} \int_{0}^{1} v_{t}^{2}(x, t) d x \leq \Lambda, \quad \int_{0}^{T} \int_{0}^{1} v_{x t}^{2} d x d t \leq \Lambda, \quad \max _{[0, T]} \int_{0}^{1} v_{x x}^{2}(x, t) d x \leq \Lambda .
$$

We now want to bound derivatives of $\theta$. We have

LEMMA 2.10.

$$
\max _{[0, T]} \int_{0}^{1} \theta_{t}^{2}(x, t) d x \leq \Lambda, \quad \int_{0}^{T} \int_{0}^{1} \theta_{x t}^{2} d x d t \leq \Lambda, \quad \max _{[0, T]} \int_{0}^{1} \theta_{x x}^{2}(x, t) d x \leq \Lambda .
$$

Proof. With the help of (2.28), (2.46), (2.31), (2.33), and (2.30) we have

$$
\max _{Q_{T}}\left|v_{x}\right| \leq \Lambda, \quad \max _{Q_{T}} \theta \leq \Lambda .
$$

We differentiate formally (2.2) with respect to $t$, multiply by $\hat{e}_{\theta} \theta_{t}$, and integrate over $[0,1] \times[0, t], 0 \leq t \leq T$. Keeping in mind that $\theta_{t}$ vanishes on the boundary, we integrate by parts with respect to $x$. Utilizing (2.48), after a lengthy calculation, which is recorded in [3, Lemma 3.6] and thus need not be reproduced here, we obtain (2.47).

Proof of Theorem 2.1. We use (2.47) and the Schwarz inequality to see that $\theta(x, t)$, respectively $\theta_{x}(x, t)$, is uniformly Hölder continuous in $t$, respectively in $x$ with 
exponent $1 / 2$. A standard interpolation property [10, II, Lemma 3.1 ; or 3 , Lemma 3.3] implies that $\theta_{x}(x, t)$ is also uniformly Hölder continuous in $t$ with exponent $1 / 6$; hence, $\left\|\theta_{x}\right\| \|_{1 / 3} \leq \Lambda$. This immediately yields $\|\theta\|_{1 / 3} \leq \Lambda$. Similarly, using (2.46), we conclude that $\left\|v_{x}\right\|_{1 / 3} \leq \Lambda$ and thereby $\|v\|_{1 / 3} \leq \Lambda,\|u\| \|_{1 / 3} \leq \Lambda$.

3. Proof of Theorem 1.1. In this section we prove Theorem 1.1 with the help of the Leray-Schauder fixed point theorem which we recall here for the reader's convenience.

Theorem 3.1. Let $\mathscr{B}$ be a Banach space and $P:[0,1] \times \mathscr{B} \rightarrow \mathscr{B}$ be a map with the following properties:

(i) for any fixed $\lambda \in[0,1], P(\lambda, \cdot): \mathscr{B} \rightarrow \mathscr{B}$ is completely continuous;

(ii) for every bounded subset $\mathscr{M}$ of $\mathscr{B}$, the family of maps $P(\cdot, \chi):[0,1] \rightarrow \mathscr{B}$, $\chi \in \mathscr{M}$ is uniformly equicontinuous;

(iii) there is a bounded subset $\mathscr{M}$ of $\mathscr{B}$ such that any fixed point in $\mathscr{B}$ of $P(\lambda, \cdot), \lambda \in[0,1]$, is contained in $\mathscr{M}$; .

(iv) $P(0, \cdot)$ has precisely one fixed point in $\mathscr{B}$.

Then, $P(1, \cdot)$ has at least one fixed point in $\mathscr{B}$.

For our purposes, $\mathscr{B}$ will be the Banach space of functions $\{u(x, t), v(x, t)$, $\theta(x, t)\}$ on $Q_{T}$ with $u, v, v_{x}, \theta, \theta_{x}$ in $C^{1 / 3,1 / 6}\left(Q_{T}\right)$ with norm

$\left\|\left|(u, v, \theta)\left\|_{\mathscr{B}}:=|| u\left|\left\|_{1 / 3}+|||v|\right\|_{1 / 3}+\right||| \theta\right\|_{1 / 3}+\left\|\left|v_{x}\right|\right\|_{1 / 3}+\left\|\theta_{x}\right\|_{1 / 3}\right.\right.$.

For $\lambda \in[0,1]$ we define $P(\lambda, \cdot)$ as the map that carries $\{\tilde{u}, \tilde{v}, \tilde{\theta}\} \in \mathscr{B}$ into $\{u, v, \theta\} \in \mathscr{B}$ by solving the (linearized) system

$$
\begin{gathered}
u_{t}-v_{x}=0, \\
v_{t}-\tilde{\mu}(\tilde{u}) v_{x x}+\left(\tilde{p}_{u}(\tilde{u}, \tilde{\theta})+\tilde{\mu}_{u}(\tilde{u}) \tilde{v}_{x}\right) u_{x}=-\tilde{p}_{\theta}(\tilde{u}, \tilde{\theta}) \tilde{\theta}_{x}, \\
\tilde{e}_{\theta}(\tilde{u}, \tilde{\theta}) \theta_{t}-\tilde{\kappa}(\tilde{u}, \tilde{\theta}) \theta_{x x}-\tilde{\kappa}_{u}(\tilde{u}, \tilde{\theta}) \tilde{\theta}_{x} u_{x}=-\tilde{\theta} \tilde{p}_{\theta}(\tilde{u}, \tilde{\theta}) \tilde{v}_{x}+\tilde{\mu}(\tilde{u}) \tilde{v}_{x}^{2}+\tilde{\kappa}_{\theta}(\tilde{u}, \tilde{\theta}) \tilde{\theta}_{x}^{2}
\end{gathered}
$$

with boundary and initial conditions

$$
\begin{gathered}
\left(-\tilde{p}(\tilde{u}, \tilde{\theta})+\tilde{\mu}(\tilde{u}) v_{x}\right)(1, t)=-\gamma \tilde{v}(1, t), \quad\left(-\tilde{p}(\tilde{u}, \tilde{\theta})+\tilde{\mu}(\tilde{u}) v_{x}\right)(0, t)=\gamma \tilde{v}(0, t), \\
\theta(1, t)=\theta(0, t)=T_{0}, \\
u(x, 0)=(1-\lambda) \eta_{0}+\lambda u_{0}(x), \quad v(x, 0)=\lambda v_{0}(x), \\
\theta(x, 0)=(1-\lambda) T_{0}+\lambda \theta_{0}(x),
\end{gathered}
$$

where $\eta_{0}$ is defined by (1.12). To solve (3.1)-(3.3) for arbitrary $\tilde{u}, \tilde{\theta} \in C^{1 / 3,1 / 6}\left(Q_{T}\right)$, we have to define $\hat{e}, \hat{p}, \hat{\mu}, \hat{\kappa}$ on $\mathbb{R}^{2}$. Recalling that a smooth solution $u(x, t)$ of the original system (1.1), (1.5)-(1.6) will be bounded from below by $\bar{u}$ and from above by $\bar{U}$ (cf. Lemma 2.3), we construct $C^{2}$-smooth functions $\tilde{e}(u, \theta), \tilde{p}(u, \theta), \tilde{\mu}(u)$, $\tilde{\kappa}(u, \theta)$ on $\mathbb{R}^{2}$ which coincide with $\hat{e}(u, \theta), \hat{p}(u, \theta), \hat{\mu}(u), \hat{\kappa}(u, \theta)$ for $\bar{u} \leq u \leq \bar{U}$, $0 \leq \theta<\infty$, and which satisfy the following conditions:

$$
\begin{gathered}
\tilde{\mu}(u) \geq \tilde{\mu}_{0}>0, \quad \tilde{e}_{\theta}(u, \theta), \\
\tilde{\kappa}(u, \theta) \geq \tilde{\nu}>0 \quad \text { for }-\infty<u<\infty,-\infty<\theta<\infty .
\end{gathered}
$$


Under these assumptions (3.1)-(3.3) will have a unique solution not only in $\mathscr{B}$ but in a better function space. More precisely,

LEMmA 3.2. There is a unique solution $\{u(x, t), v(x, t), \theta(x, t)\}$ of (3.1)-(3.3) such that $u, u_{t}, u_{x}, v, v_{t}, v_{x}, v_{x x}, \theta, \theta_{t}, \theta_{x}, \theta_{x x}$ are all in $C^{\beta, \beta / 2}\left(Q_{T}\right)$ with $\beta=$ $\min \{\alpha, 1 / 3\}$, and the $C^{\beta, \beta / 2}\left(Q_{T}\right)$ norms of these functions can be a priori bounded in terms of $C$ (depending only on $\tilde{\mu}_{0}, \tilde{\nu}, T$, and on the $C^{\beta, \beta / 2}\left(Q_{T}\right)$ norms of the coefficients) times the norms of the initial data and the right-hand sides of (3.1).

The existence of a solution to (3.1)-(3.3) can be established by the method of continuity (see [4]) which connects (3.1) to a system without $u_{x}$-terms in $(3.1)_{2}$ and $(3.1)_{3}$. The uniqueness is obvious. The a priori estimate in Lemma 3.2 follows from the classical Schauder-Friedman estimate (cf. $[4,10]$ ).

Applying Lemma 3.2 we see that any solution to (3.1)-(3.3) will be in the space indicated in Lemma 3.2. It then follows from the interpolation property [10, II, Lemma 3.1; or 3, Lemma 3.3] that the solution $\{u, v, \theta\}$ to (3.1)-(3.3) is in a Hölder space which is compactly imbedded in $\mathscr{B}$ (in fact $u, v, v_{x}, \theta, \theta_{x} \in C^{1,1 / 2}\left(Q_{T}\right)$ ). Thus, $P(\lambda, \cdot): \mathscr{B} \rightarrow \mathscr{B}$ is not only well defined, but also completely continuous as required in (i) of Theorem 3.1. Using the a priori estimate in Lemma 3.2, we can easily show that the family $P(\cdot,\{\tilde{u}, \tilde{v}, \tilde{\theta}\}):[0,1] \rightarrow \mathscr{B}$, with $\{\tilde{u}, \tilde{v}, \tilde{\theta}\}$ in any fixed bounded subset of $\mathscr{B}$, is uniformly equicontinuous, so that (ii) of Theorem 3.1 holds.

To show (iii) we note that any fixed point $\{u, v, \theta\}$ of $P$ will solve the system $(1.1)_{1},(2.1),(2.2),(3.3),(1.6)$ with $\hat{e}, \hat{p}, \hat{\mu}$, and $\hat{\kappa}$ replaced by $\tilde{e}, \tilde{p}, \tilde{\mu}$, and $\tilde{\kappa}$. Recalling that $\tilde{e}=\hat{e}, \tilde{p}=\hat{p}, \tilde{\mu}=\hat{\mu}, \tilde{\kappa}=\hat{\kappa}$ for $\bar{u} \leq u \leq \bar{U}$ and $\theta \geq 0$, Lemmas 2.1 and 2.3 imply that $\theta>0$ and $\bar{u} \leq u \leq \bar{U}$; thus, $\tilde{e}, \tilde{p}, \tilde{\mu}, \tilde{\kappa}$ coincide with $\hat{e}, \hat{p}, \hat{\mu}, \hat{\kappa}$. Hence, any fixed point of $P$ will be a solution of the original system (1.1), (3.3), (1.6), where the assumptions (1.15)-(1.17) are satisfied, and (iii) thus follows from Theorem 2.1 .

To verify (iv) we easily see by virtue of (1.12) that $u(x, t)=\eta_{0}, v(x, t)=0$, $\theta(x, t)=T_{0}$ is a fixed point of $P(0, \cdot)$. This solution is unique. The uniqueness of any fixed point of $P(\lambda, \cdot)$ for $\lambda \in[0,1]$ can be shown in a standard fashion which is outlined in [3, Lemma 3.8]. Hence, Theorem 3.1 implies that $P(1, \cdot)$ has at least one fixed point; this point is unique, i.e., there is a unique solution of (1.1), (1.5), (1.6) on $[0,1] \times[0, T]$ in the function class indicated in Lemma 3.2. To complete the proof of Theorem 1.1 it remains to show that the solution of (1.1), (1.5), (1.6) has derivatives not only in $C^{\beta, \beta / 2}$ with $\beta=\min \{\alpha, 1 / 3\}$ but also in $C^{\alpha, \alpha / 2}$. This can be done by noting that $u, v, v_{x}, \theta, \theta_{x}$ are in $C^{1,1 / 2}\left(Q_{T}\right)$ and by another application of Lemma 3.2 with $\beta=\min \{\alpha, 1\}$. This completes the proof of Theorem 1.1.

Acknowledgment. The author was supported by the Sonderforschungsbereich 256 of the Deutsche Forschungsgemeinschaft at the University of Bonn. 


\section{REFERENCES}

[1] C. M. Dafermos, Contemporary issues in the dynamic behavior of continuous media, Lefschetz Center for Dynamical Systems Report, Brown Univ., Providence, RI, 1985

[2] __ Global smooth solutions to the initial boundary value problem for the equations of onedimensional nonlinear thermoviscoelasticity, SIAM J. Math. Anal. 13, 397-408 (1982)

[3] C. M. Dafermos and L. Hsiao, Global smooth thermomechanical processes in one-dimensional nonlinear thermoviscoelasticity, Nonlinear Anal. T.M.A. 6, 435-454 (1982)

[4] A. Friedman, Partial Differential Equations of Parabolic Type, Prentice-Hall, Englewood Cliffs, NJ, 1964

[5] S. Kawashima and T. Nishida, Global solutions to the initial value problem for the equations of one-dimensional motion of viscous polytropic gases, J. Math. Kyoto Univ. 21, 825-837 (1981)

[6] B. Kawohl, Global existence of large solutions to initial boundary value problems for a viscous, heat-conducting, one-dimensional real gas, J. Differential Equations 58, 76-103 (1985)

[7] A. V. Kazhykhov, Sur la solubilité globale des problèmes monodimensionnels aux valeurs initialeslimitées pour les équations d'un gaz visqueux et calorifere, C. R. Acad. Sci. Paris Sér. A 284, 317-320 (1977)

[8] A. V. Kazhikhov and V. V. Shelukhin, Unique global solution with respect to time of initial boundary value problems for one-dimensional equations of a viscous gas, J. Appl. Math. Mech. 41, 273$282(1977)$

[9] J. U. Kim, Global existence of solutions of the equations of one-dimensional thermoviscoelasticity with initial data in $B V$ and $L^{1}$, Ann. Scuola Norm. Sup. Pisa Cl. Sci. (4) 10, 357-427 (1983)

[10] O. A. Ladyzenskaya, V. A. Solonnikov, and N. N. Ural'ceva, Linear and Quasilinear Equations of Parabolic Type, transl. Math. Monographs, vol. 23, Amer. Math. Soc., Providence, RI, 1968

[11] T. Nagasawa, On the one-dimensional motion of the polytropic ideal gas nonfixed on the boundary, J. Differential Equations 65, 49-67 (1986)

[12] _ On the outer pressure problem of the one-dimensional polytropic ideal gas, Japan J. Appl. Math. 5, 53-85 (1988)

[13] T. Nishida, Equations of motion of compressible viscous fluids, Patterns and Waves-Qualitative Analysis of Nonlinear Differential Equations (T. Nishida, M. Mimura, and H. Fujii, eds.), Kinokuniya/North-Holland, Tokyo/Amsterdam, 1986

[14] M. Okada and S. Kawashima, On the equations of one-dimensional motion of compressible viscous fluids, J. Math. Kyoto Univ. 23, 55-71 (1983)

[15] M. H. Protter and H. F. Weinberger, Maximum Principles in Differential Equations, Prentice-Hall, Englewood Cliffs, NJ, 1967

[16] S. Zheng and W. Shen, Global smooth solutions to the Cauchy problem of equations of onedimensional nonlinear thermoviscoelasticity, J. Partial Differential Equations 2, 26-38 (1989) 